\title{
The Thermodynamics and Kinetics of High-Entropy Alloys
}

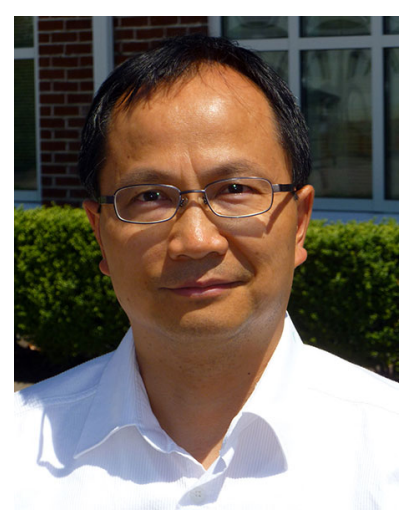

M.C. Gao

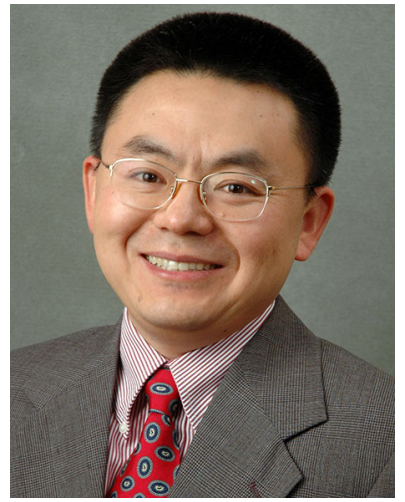

J.-C. Zhao

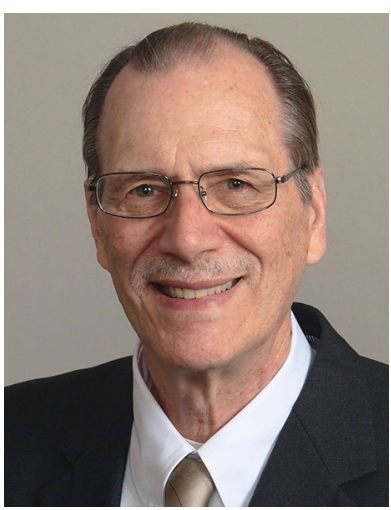

J.E. Morral

The first ten articles in this issue of the Journal of Phase Equilibria and Diffusion are on the topic of high-entropy alloys (HEAs). These alloys have attracted significant attention in recent years for both scientific curiosity and potential applications (High-Entropy Alloys: Fundamentals and Applications (2016). 1st ed. M.C. Gao, J.W. Yeh, P.K. Liaw, Y. Zhang (eds.), Springer Cham, Switzerland). Different from traditional alloys that focus on an edge or corner of a phase diagram, HEAs have compositions located at or near the center of five or more-component composition spaces. That is where the ideal configurational entropy is highest. While attractive physical, mechanical, and functional properties have been reported for a variety of HEAs, there are still challenges to fully understand their behavior and properties. 
Professor J.-W. Yeh first hypothesized the four core effects of HEAs: (1) high entropy effect for thermodynamics, (2) sluggish diffusion effect for kinetics, (3) severe lattice distortion effect for structure, and (4) a "cocktail" effect for properties (J.W. Yeh, Recent progress in highentropy alloys, Annales de Chimie-Science des Materiaux, 2006, 31(6), pp 633-648). The cocktail effect here means that the properties of HEAs may deviate from the rule-ofmixtures. Nearly all of Yeh's core effects, including entropy, diffusion, structure, and properties, are mainstays of the Journal of Phase Equilibria and Diffusion. Therefore we invited researchers to submit papers to JPED for this special issue with a focus on HEAs.

The objective of the special focus is to gather and disseminate, in a timely fashion, recent progress on the thermodynamics and kinetics of HEAs. In addition, it is meant to engage readers, reviewers, and editors of JPED in this growing field. Within the past ten years the number of papers on HEAs has grown from about 100 to 400 papers/ year according to Scopus. Of these papers, those on thermodynamics have grown from about one paper/year to 20/year, while on diffusion from 3 papers/year to 30/year In the current issue, three papers are on calculating phase diagrams, three on interdiffusion, two on structure, and two on solid solutions.

We expect the yearly number of papers on phase equilibria and kinetics to continue to grow. One reason is that there has been little fundamental work in the past on alloys that contain five or more multiple principal elements (e.g. HEA alloys). Many studies on HEAs to date have been exploratory experimental work or prediction work based on either ideal/regular solution models or databases validated for only two or three principal elements. Recently thermodynamic databases specifically developed for HEAs have become available, for example as described by Erik Holmstrom et al. in this issue. However the validity of extrapolating data and concepts from the three-dimensional space of ternaries to the hyperspace of HEAs remains to be seen.

In our view the field of HEA is still in the early stages of development. Like all true explorations into the unknown, we can plan where we are going, but not foretell the destination. That is what makes this field so intriguing.

\section{M.C. Gao}

National Energy Technology Laboratory/AECOM, OR, email: Michael.Gao@netl.doe.gov

\section{J.-C. Zhao}

Associate Editor

Journal of Phase Equilibria and Diffusion

The Ohio State University Columbus, $\mathrm{OH}$, email: zhao.199@osu.edu

\section{J.E. Morral}

Editor-in-Chief

Journal of Phase Equilibria and Diffusion

The Ohio State University, Columbus, $\mathrm{OH}$, email: jemorral@gmail.com 